\title{
El desarrollo de competencias profesionales en el Practicum de maestros. La visión de sus protagonistas
}

\section{The development of professional skills in the Practicum of teachers. The vision of its protagonists}

\author{
DEL ARCO, Isabel ${ }^{1}$ \\ GAIRÍN, Joaquín ${ }^{2}$ \\ ARMENGOL, Carme ${ }^{3}$ \\ Resumen \\ El objetivo del estudio es analizar el desarrollo competencial que promueve el Practicum en la formación \\ de maestros, desde la visión de sus protagonistas. Bajo una metodología mixta y secuencial, se \\ triangulan datos cuantitativos obtenidos de cuestionarios con datos de entrevistas y grupos de \\ discusión. Los resultados evidencian que hay un desarrollo competencial en cuanto al conocimiento \\ práctico del funcionamiento y gestión del aula, pero la función tutorial no forma parte substancial del \\ desarrollo profesional que potencia este momento formativo. \\ Palabras clave: practicum, competencia profesional, formación inicial, identidad profesional
}

\begin{abstract}
The objective of the study is to analyze the competence development promoted by the Practicum in teacher training, from the perspective of its protagonists. Under a mixed and sequential methodology, quantitative data obtained from questionnaires are triangulated with data from interviews and discussion groups. The results show that there is a competence development in terms of practical knowledge of the operation and management of the classroom, but the tutorial function is not a substantial part of the professional development that enhances this formative moment

Key words: practicum, professional competence, initial training, professional identity
\end{abstract}

\section{Introducción}

El Practicum en la formación inicial de maestros es un componente esencial y tiene un peso significativo en los actuales planes de estudios de grado en toda Europa. Bajo modelos concurrentes o consecutivos, es decir, a lo largo de tres o cuatro cursos o al final de la formación, la distribución porcentual de los 25 países europeos analizados en el Libro Blanco (ANECA, 2004) confirma que más del 50\% optan por un modelo de formación con

\footnotetext{
1 Profesora Titular de Universidad. Departamento de Pedagogía de la Facultad de Educación, Psicología y Trabajo Social de la Universidad de Lleida.Cataluña. España. Correo electrónico: Isabel.delarco@udl.cat

${ }^{2}$ Catedrático de Universidad. Departamento de Pedagogía Aplicada de la Facultad de Educación de la Universidad Autónoma de Barcelona .Cataluña. España. Correo electrónico: Joaquin.Gairín@uab.cat

${ }^{3}$ Profesora Titular de Universidad. Departamento de Pedagogía Aplicada de la Facultad de Educación de la Universidad Autónoma de Barcelona. Cataluña. España. Correo electrónico: carme.armengol@uab.cat
} 
un mínimo de 60 ECTS (European Credit Transder System). Su inclusión en los programas formativos reconoce explícitamente que, además del conocimiento técnico y científico, es indispensable un conocimiento práctico que permita una aproximación a la realidad donde ejercerán los futuros profesionales.

El Practicum constituye un momento clave para movilizar conocimientos, habilidades y actitudes que resultan difíciles de trabajar en el contexto universitario, al permitir una interacción con situaciones reales y en escenarios propios de la profesión (Cabrerizo, Rubio y Castillo, 2010). Sin embargo, esta interacción con situaciones reales ha de ir más allá del aprender a enseñar haciendo y debe ser una consecuencia directa de un proceso de reflexión sobre la acción. Solamente ante este diálogo con tu yo reflexivo (Villar, 1998:270), el aprendiz será consciente de cómo y por qué hace las cosas, de posibles alternativas y de sus consecuencias.

Uno de los retos importantes es definir cuáles son las competencias que deben desarrollarse en el Practicum y las actividades que permiten su consecución. Muchos autores han hecho aportaciones al tema: Gairín, Armengol, del Arco, Muñoz y Rodríguez-Gómez, (2013) y González Sanmamed (2001) analizan las competencias profesionales que el estudiante en prácticas debe movilizar para el ejercicio de las tareas de maestro. Marhuenda, Bernad y Navas (2010) hablan de competencias de empleabilidad para referirse al conjunto de capacidades esenciales para aprender y desarrollarse eficazmente en el lugar de trabajo, incluyendo capacidades de comunicación y relación interpersonal, resolución de problemas y manejo de procesos organizacionales y de organización de los propios comportamientos en función de los requerimientos del puesto de trabajo.

El comité mixto OIT/UNESCO realizó diversos informes sobre la situación del personal docente y entre los que destacamos los del 2006 y 2008 ya que en ellos recomendó examinar los planes de formación inicial para que sea viable la capacitación de docentes reflexivos, innovadores, con habilidades para responder eficientemente en entornos cambiantes. Para ello, propone elaborar marcos de competencia profesional bajo los que se diseñen los planes de estudio y donde se establezcan las prácticas bajo la supervisión de profesores cualificados.

También se han realizado otros estudios e informes, destacando los realizados por Eurydice (2002, 2004, 2005) sobre la profesión docente en Europa, por la OCDE a través de los diferentes informes TALIS desde el primer ciclo en el 2008 hasta la actualidad. En todos ellos se trabaja el perfil, las competencias profesionales y el modelo de docente en la Europa del siglo XXI. Se coincide en afirmar que en los últimos años se han ido incorporando nuevas competencias profesionales a los maestros en consonancia con las nuevas circunstancias sociales $y$ culturales: idiomas, multiculturalidad, cuestiones de género y de convivencia, diversidad del alumnado y nuevas tecnologías, entre otras.

Todos los estudios e informes citados consideran que las competencias incluyen saberes o conocimientos (saber), habilidades, conocimientos prácticos y destrezas (saber hacer), valores, actitudes y compromisos personales (saber ser y estar) y su uso funcional e integrado en contextos variados para resolver los retos profesionales. En resumen, estos informes coinciden en establecer una serie de competencias propias de lo que podemos decir sería el modelo europeo de docente, clasificando 10 competencias profesionales en cinco ámbitos (tabla 1): 
Tabla 1

Competencias profesionales del docente según el modelo europeo (elaboración propia)

\begin{tabular}{|c|c|c|}
\hline Ámbitos & Competencias & Concreción \\
\hline SABER & $\begin{array}{l}\text { Científica: gestión del conocimiento en el área } \\
\text { de la educación y del resto de áreas curriculares. }\end{array}$ & $\begin{array}{l}\text { 1.1. Conocimiento critico de aportaciones en el área } \\
\text { de educación. } \\
\text { 1.2. Conocimiento crítico de aportaciones en las } \\
\text { áreas, materias y módulos curriculares. } \\
\text { 1.3. Gestión del conocimiento individual y colectivo. }\end{array}$ \\
\hline SABER SER & $\begin{array}{l}\text { Intra-personal: forma de ser y forma de tratar a } \\
\text { los demás, usando habilidades personales, para } \\
\text { la acción tutorial, la orientación y promoción de } \\
\text { los valores, etc. }\end{array}$ & $\begin{array}{l}\text { 2.1. Habilidades personales. } \\
\text { 2.2. Acción tutorial. } \\
\text { 2.3. Orientación. } \\
\text { 2.4. Gestión y promoción de valores. }\end{array}$ \\
\hline $\begin{array}{c}\text { SABER } \\
\text { HACER QUE }\end{array}$ & $\begin{array}{l}\text { Didáctica: saber enseñar y gestionar todo el } \\
\text { proceso de enseñanza-aprendizaje. Se hace } \\
\text { operativa en las programaciones didácticas, con } \\
\text { las estrategias de atención a la diversidad, la } \\
\text { gestión del aula, recursos y materiales } \\
\text { didácticos, los procesos de evaluación, etc. } \\
\text { Organización y gestión del trabajo: relacionada } \\
\text { con la normativa, la planificación, la } \\
\text { coordinación y la gestión de la calidad del centro } \\
\text { educativo. } \\
\text { Gestión de la convivencia: habilidades } \\
\text { desarrolladas para una convivencia con los } \\
\text { demás, mediante la promoción, mediación y } \\
\text { control. }\end{array}$ & $\begin{array}{l}\text { 3.1. Programación. } \\
\text { 3.2. Didácticas específicas de áreas, materias y } \\
\text { módulos. } \\
\text { 3.3. Metodología y actividades. } \\
\text { 3.4. Atención a la diversidad. } \\
\text { 3.5. Gestión del aula (espacios de aprendizaje). } \\
\text { 3.6. Recursos y materiales. } \\
\text { 3.7. Evaluación. } \\
\text { 4.1. Normativa. } \\
\text { 4.2. Organización, planificación, coordinación } \\
\text { (desempeño de plazas específicas). } \\
\text { 4.3. Gestión de la calidad. } \\
\text { 5.1. Promoción de la convivencia. } \\
\text { 5.2. Mediación, resolución de conflictos. } \\
\text { 5.3. Control de la convivencia. }\end{array}$ \\
\hline $\begin{array}{l}\text { SABER } \\
\text { HACER } \\
\text { COMO }\end{array}$ & $\begin{array}{l}\text { Trabajo en equipo: relacionada con el trabajo } \\
\text { colaborativo con objetivos comunes. } \\
\text { Innovación y mejora: procesos de gestión del } \\
\text { cambio, saber hacer diagnosis, hacer nuevas } \\
\text { propuestas, implementarlas y evaluarlas para } \\
\text { conocer el impacto sobre la mejora producida. } \\
\text { Comunicativa y lingüística: intercambio del } \\
\text { conocimiento, ideas, pensamientos, emociones } \\
\text { y sentimientos y su transferencia. Está } \\
\text { relacionada con la gestión de la información, la } \\
\text { transparencia y la comunicación haciendo uso } \\
\text { de cualquier lenguaje. } \\
\text { Digital: conocimiento de las tecnologías de la } \\
\text { información y la comunicación, y del uso } \\
\text { didáctico de éstas. }\end{array}$ & $\begin{array}{l}\text { 6.1. Actitudes de cooperación y colaboración. } \\
\text { 6.2. Participación e implicación en Proyectos } \\
\text { comunes. } \\
\text { 6.3. Técnicas de trabajo en grupo. } \\
\text { 6.4. Toma de decisiones. Asunción de } \\
\text { responsabilidades. } \\
\text { 7.1. Gestión del cambio. } \\
\text { 7.2. Investigación. } \\
\text { 7.3. Diagnóstico y evaluación. } \\
\text { 7.4. Realización y ejecución de propuestas. } \\
\text { 8.1. Gestión de la información y transparencia. } \\
\text { 8.2. Expresión y comunicación. } \\
\text { 8.3. Destrezas comunicativas en lengua propia. } \\
\text { 8.4.Destrezas lingüística-comunicativas en lenguas } \\
\text { extranjeras } \\
\text { 9.1. Conocimiento de las tecnologías. } \\
\text { 9.2. Uso didáctico de las mismas. } \\
\text { 9.3. Desarrollo profesional y de gestión. } \\
\text { 9.4. Aspectos actitudinales y éticos. }\end{array}$ \\
\hline $\begin{array}{l}\text { SABER } \\
\text { ESTAR }\end{array}$ & $\begin{array}{l}\text { Social-relacional: relaciones entre las personas y } \\
\text { participación en comunidad. }\end{array}$ & $\begin{array}{l}\text { 10.1. Equidad. } \\
\text { 10.2. Habilidades sociales. } \\
\text { 10.3. Habilidades relacionales. } \\
\text { 10.4. Gestión de la participación. }\end{array}$ \\
\hline
\end{tabular}


Más concretamente, la Orden ECl/3857/2007 establece las competencias a alcanzar en el período de prácticas en los siguientes términos:

- Adquirir un conocimiento práctico del aula y de la gestión de la misma.

- Conocer y aplicar los procesos de interacción y comunicación en el aula y dominar las destrezas y habilidades sociales necesarias para fomentar un clima de aula que facilite el aprendizaje y la convivencia.

- Controlar y hacer el seguimiento del proceso educativo y en particular el de enseñanza-aprendizaje mediante el dominio de las técnicas y estrategias necesarias.

- Relacionar teoría y práctica con la realidad del aula y del centro.

- Participar en la actividad docente y aprender a saber hacer, actuando y reflexionando desde la práctica. Participar en las propuestas de mejora en los distintos ámbitos de actuación que se puedan establecer en un centro.

- Regular los procesos de interacción y comunicación en grupos de estudiantes de 6-12 años.

- Conocer formas de colaboración con los distintos sectores de la comunidad educativa y del entorno social. Aunque se trata de una explicitación muy genérica de las competencias a trabajar, su identificación ayuda a establecer un marco de referencia para que tutores de las escuelas y de la universidad compartan el conocimiento sobre las competencias que deben desarrollar los aspirantes a maestro.

\section{Objetivos}

El objetivo general es analizar la visión de los diversos participantes sobre el desarrollo competencial que promueve el Practicum y las competencias profesionales que más desarrolla. Así, interesa conocer las opiniones de: i) estudiantes, ii) maestros tutores de las escuelas, iii) tutores de la universidad y iv) responsables de la coordinación del Practicum en ambas instituciones, sobre el desarrollo competencial durante el Practicum, para averiguar cuáles son las competencias que más se trabajan y cuáles las que quedan en un segundo término

\section{Metodología}

La metodología de la investigación corresponde a lo que Creswell (2009) califica, dentro de las metodologías mixtas, como una aproximación secuencial. Primero se realiza un estudio cuantitativo (mediante cuestionarios, en nuestro caso) para posteriormente aplicar una metodología cualitativa que completa y contrasta los resultados (mediante estudio de casos, análisis documental y grupos de discusión).

El uso de diferentes instrumentos e informantes para la recogida de datos permite la contrastación y su triangulación. Mientras que los datos cuantitativos permiten "vincular conceptos abstractos con indicadores empíricos" (Hernández, Fernández-Collado y Baptista, 2008: 345), los cualitativos permiten "comprender en profundidad fenómenos educativos y sociales" (Sandín, 2003: 123).

\subsection{Participantes}

Se contactaron un total de 501 estudiantes de Practicum, 370 tutores de escuela, 89 tutores de facultad y 26 coordinadores, de los grados de Infantil y Primaria de la Universitat Autònoma de Barcelona (UAB, en adelante) y de la Universitat de Lleida (UdL, en adelante) (tabla 2), vinculados a las actividades de Practicum que se realizaban en las escuelas seleccionadas. 
Tabla 2

Tipología de informantes

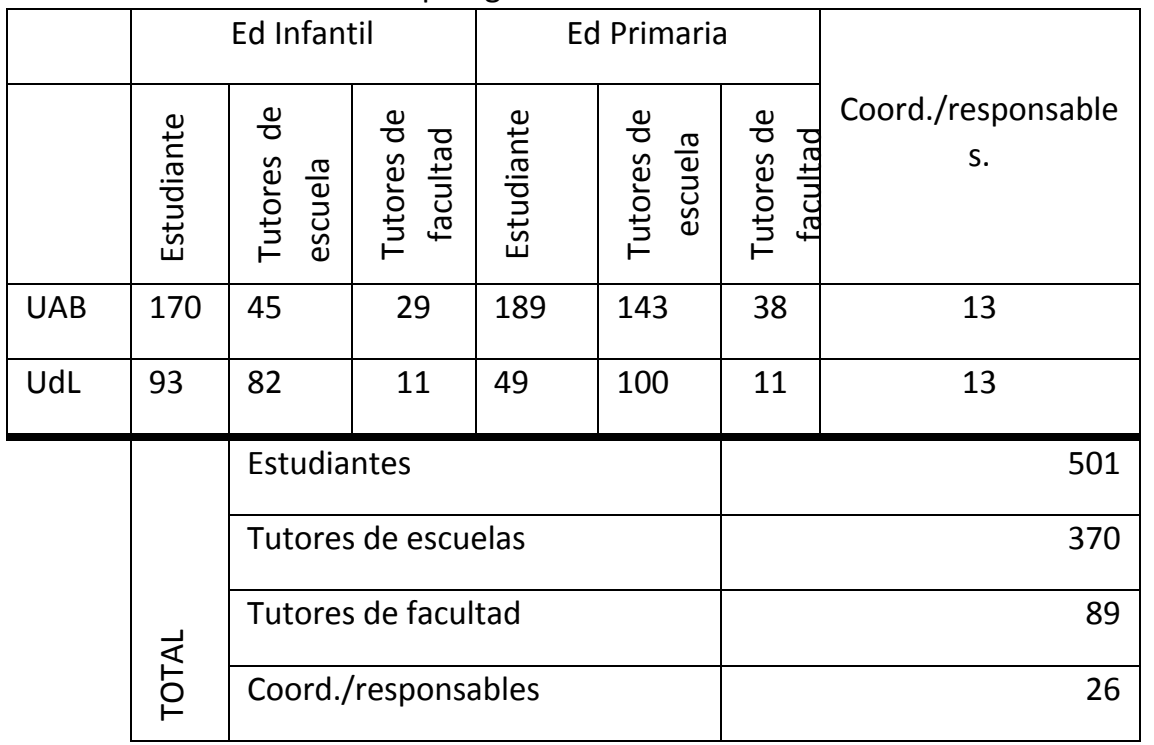

Se estableció una distribución de escuelas que permitiera recoger datos de diferente naturaleza (tabla 3): escuelas de prácticas, concertadas, rurales, centros de nueva creación, de especial dificultad y singulares. Su selección tuvo en cuenta criterios de accesibilidad y compromiso con el estudio.

Tabla 3

Escuelas participantes

\begin{tabular}{|c|c|c|c|}
\hline $\mathrm{N}_{\text {TIPOLOGIA }}$ UNIVERSIDAD & UAB & UdL & TOTAL \\
\hline Escuela de prácticas & 1 & 1 & 2 \\
\hline Concertadas & $\begin{array}{c}3 \\
\text { (1 religiosa y } 2 \\
\text { cooperativa/ } \\
\text { laica) }\end{array}$ & $\begin{array}{c}2 \\
\text { (1 religiosa y } 1 \\
\text { laica) }\end{array}$ & 5 \\
\hline ZER & 0 & 1 & 1 \\
\hline Rural completa & 0 & 1 & 1 \\
\hline Nueva creación (0-10 años) & 1 & 1 & 2 \\
\hline Centros de especial dificultad & 1 & 1 & 2 \\
\hline Régimen ordinario/Singulares & 2 & 1 & 3 \\
\hline TOTAL & $8^{*}$ & $8^{*}$ & 16 \\
\hline
\end{tabular}

\subsection{Instrumentos}

- Tres cuestionarios creados y validados para el estudio, uno para cada perfil de informantes: estudiantes, tutores de escuela y tutores de facultad. Los cuestionarios se estructuraron teniendo en cuenta las dimensiones de competencias: Aplicación del conocimiento a la práctica (CP), de gestión dinámica del aula y funcionamiento de aula (CDA) y de relaciones y formas de trabajo (FT), recogidas en el tabla 4. 
Tabla 4

Competencias analizadas según dimensiones

\begin{tabular}{|c|c|}
\hline \multicolumn{2}{|r|}{ Dimensiones } \\
\hline CDA & $\begin{array}{l}\text { Con las estancias en el centro se adquiere un conocimiento práctico del aula y de la gestión de la misma (por } \\
\text { ejemplo, organización de los alumnos, organización del tiempo, organización de los espacios, etc.) }\end{array}$ \\
\hline CDA & El estudiante conoce y aplica los procesos de interacción y comunicación en el aula \\
\hline CDA & $\begin{array}{l}\text { El estudiante domina las destrezas y habilidades sociales necesarias para fomentar un clima de aula que } \\
\text { facilite el aprendizaje y la convivencia }\end{array}$ \\
\hline CDA & El estudiante identifica variables que pueden incidir en el clima y la cultura del centro como organización \\
\hline CDA & $\begin{array}{l}\text { El estudiante controla y hace el seguimiento del proceso de enseñanza / aprendizaje mediante el dominio de } \\
\text { las técnicas y estrategias necesarias }\end{array}$ \\
\hline $\mathrm{CP}$ & El estudiante relaciona la teoría con la realidad del aula \\
\hline $\mathrm{CP}$ & El estudiante relaciona la teoría con la realidad de la escuela \\
\hline $\mathrm{CP}$ & $\begin{array}{l}\text { El estudiante conoce diferentes propuestas metodológicas / buenas prácticas docentes y las vincula con su } \\
\text { conocimiento teórico }\end{array}$ \\
\hline $\mathrm{CP}$ & El estudiante participa en la actividad docente \\
\hline $\mathrm{CP}$ & El estudiante aprende a saber hacer, actuando y reflexionando desde la práctica \\
\hline $\mathrm{FT}$ & $\begin{array}{l}\text { El estudiante participa en las propuestas de mejora en los distintos ámbitos de actuación que se puedan } \\
\text { establecer en el centro }\end{array}$ \\
\hline CFA & El estudiante regula los procesos de interacción y comunicación de los grupos \\
\hline FT & $\begin{array}{l}\text { El estudiante conoce las formas de colaboración establecidas entre los diferentes sectores de la comunidad } \\
\text { educativa y del entorno social }\end{array}$ \\
\hline FT & El estudiante conoce el desarrollo tutorial y cómo se hacen los contactos / entrevistas con los padres-madres \\
\hline CDA & $\begin{array}{l}\text { Con las estancias en el centro se adquiere un conocimiento práctico del aula y de la gestión de la misma (por } \\
\text { ejemplo, organización de los alumnos, organización del tiempo, organización de los espacios, etc.) }\end{array}$ \\
\hline
\end{tabular}

- Estudio de casos en las 16 escuelas, que supuso entrevistas en profundidad a estudiantes que realizaban las prácticas en estos centros, a tutores de escuela y facultad y a coordinadores/responsables (en total 101 informantes). En las entrevistas, se preguntó sobre el conocimiento práctico del aula y el conocimiento sobre la acción tutorial que se desarrollaba, indagando en qué aspecto se incidía más.

- Análisis documental de 28 memorias de prácticas de diferentes cursos del Practicum de cada universidad y titulación.

- Grupo de discusión, uno por universidad donde participaron los responsables de la coordinación, diseño y desarrollo del Practicum. En total 10 informantes por universidad entre coordinadores de las facultades, representantes de dirección de las escuelas y representante de la Administración Educativa con atribuciones en las prácticas. El guion del grupo de discusión queda recogido en la tabla 5.

\section{Tabla 5}

Guion del grupo de discusión

ADQUISICIÓN: Estudiantes y tutores consideran que la adquisición de competencias se produce progresivamente. ¿Está de acuerdo? ¿Qué competencias cree que se desarrollan de manera más notable durante el Practicum? ¿Cree que hay competencias que sólo se pueden desarrollar en los Practicums los últimos cursos del Grado?

COMPETENCIAS QUE MÁS SE TRABAJAN: Estudiantes y tutores coinciden en que es la gestión del aula la competencia que más se trabajan desde el Practicum. ¿Está de acuerdo? ¿Por qué cree que es la competencia más desarrollada durante el Practicum?

COMPETENCIAS QUE MENOS SE TRABAJAN: Estudiantes y tutores de facultad consideran que la acción tutorial es la competencia que menos se trabaja desde el Practicum. ¿Está de acuerdo? ¿Por qué cree que es la competencia menos desarrollada durante el Practicum? 


\subsection{Método}

Para el análisis de los cuestionarios, se ha utilizado el paquete estadístico SPSSx versión 15.0, mientras que para el análisis de datos cualitativos se ha seguido el proceso general de análisis de datos cualitativos propuesto por Miles y Huberman (1994) y revisado por Saldaña (Miles, Huberman y Saldaña, 2014). El análisis de datos ha seguido un proceso interpretativo de codificación y categorización de unidades de significado (US) de todos los datos obtenidos. El análisis ha contemplado complementariamente planteamientos de codificación deductiva e inductiva, siguiendo el Modelo simplificado de códigos de Saldaña (2009): a) identificación de las US; b) agrupación de las US en categorías; y c) surgimiento de las metacategorías/temas. Complementariamente, seguimos los principios de la técnica de comparación analítica de Coller (2000), sumando ejemplos de los diversos casos.

Los datos obtenidos por medio de cada instrumento se analizaron por separado, elaborando informes de los resultados de: cuestionarios (análisis descriptivo y de varianza), estudios de caso (análisis transversal de los casos en función de dimensiones de análisis), análisis documental de las memorias de Practicum y grupos de discusión. Una vez dispuestos los resultados obtenidos por medio de cada instrumento se procedió a la triangulación de datos.

\section{Resultados}

Los resultados obtenidos se presentan en función de tres bloques:

- Adquisición de competencias

- Competencias que más se trabajan

- Competencias que menos se trabajan

\subsection{Adquisición de competencias}

Los datos (ver tabla 6) muestran que hay cierta coincidencia entre los tres colectivos (estudiantes, tutores de escuela y tutores de facultad), en relación a las competencias que más se trabajan desde el Prácticum, con medias que oscilan entre el 3.11, en el caso de los tutores de escuela y el 3.41, en el caso de los estudiantes.

En el caso del desarrollo de competencias (ver tabla 6), es especialmente destacable la relación que se establece en los tres colectivos (.650, .760 y .585, respectivamente) entre las competencias vinculadas a la gestión de la dinámica de la aula (fomento de un clima de aula que facilite el aprendizaje y la convivencia, aplicación de procesos de interacción y comunicación, conocimiento práctico del aula y su gestión, seguimiento del proceso de enseñanza-aprendizaje) y la aplicación de conocimientos teóricos en el aula (relación de la teoría con el realidad del aula y de la escuela, aplicación de propuestas metodológicas y vinculación con su conocimiento teórico, actuación y reflexión sobre la práctica). Por otra parte, a diferencia de lo que ocurre con la satisfacción, en este caso no hay acuerdo en los tres conjuntos de datos (estudiantes, tutores de escuela y tutores de facultad) en una clara identificación de cuál es el factor más importante asociado al desarrollo de competencias. Mientras en el caso de los estudiantes, las relaciones y formas de trabajo (participación en propuestas de mejora, conocimientos sobre las formas de colaboración entre la comunidad educativa y el entorno, participación en propuestas de mejora, regulación de procesos de interacción y comunicación, acción tutorial) parecen ser las competencias más destacables, los tutores, tantos los de escuela, como los de universidad, entiende que son aquellas competencias vinculadas a la aplicación de conocimientos a la práctica las más importantes.

No existe acuerdo entre los informantes (estudiantes, tutores de escuela y tutores de facultad) en relación a la adquisición de competencias. Mientras que en el caso de los estudiantes, las relaciones y formas de trabajo 
(participación en propuestas de mejora, conocimientos sobre las formas de colaboración entre la comunidad educativa y el entorno, participación en propuestas de mejora, regulación de procesos de interacción y comunicación, acción tutorial) parecen ser las competencias más destacables, los tutores, tantos los de escuela, como los de universidad, entienden que son más importantes aquellas competencias vinculadas a la aplicación de conocimientos a la práctica.

Tabla 6

Análisis descriptivo de los factores y valores globales en la adquisición de competencias según informantes y resumen de las correlaciones entre los factores que integran la escala de desarrollo de competencias y su valor global

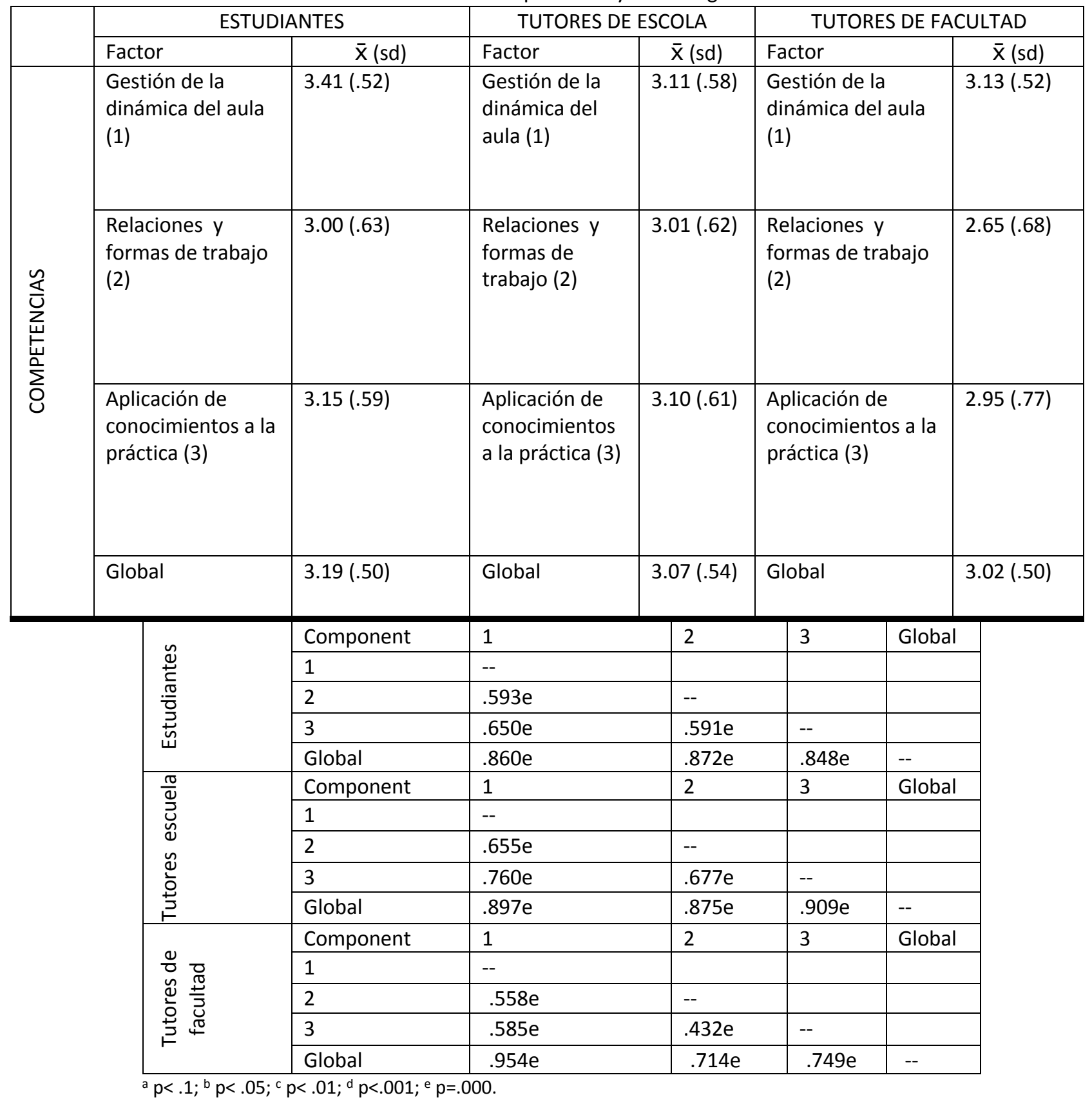

Analizando los datos globales presentados anteriormente, según el perfil de los informantes, y haciendo la prueba de homogeneidad de las varianzas (prueba de Levene) para decidir si es aplicable la prueba de F estándar 
(Toothaker, 1991; Miller, 1981) o requiere el uso de la corrección de Welch (Tomarken y Serlin, 1986) deben interpretarse de la siguiente manera:

- Si la prueba de Levene no es significativa $(p \geq .05)$, entonces se utiliza la prueba de $\mathrm{F}$ estándar para el contraste global y, en su caso, la prueba post-hoc HSD de Tukey.

- Si la prueba de Levene es significativa ( $\mathrm{p} \leq .05)$, entonces se utiliza la corrección de Welch para el contraste global y, en su caso, la prueba post-hoc GH de Games Howell.

Los estudiantes opinan que durante el Practicum desarrollan suficientemente las competencias para el ejercicio de la profesión docente. Las competencias más valoradas son: conocimiento práctico del aula y de su gestión (organización de los alumnos, del tiempo, de los espacios, etc.). También, el desarrollo de procesos de interacción y de comunicación en el aula y el dominio de la reflexión sobre la práctica docente.

Por el contrario, las competencias valoradas un grado de adquisición menor son las que hacen referencia al conocimiento de la acción tutorial y como ésta se concreta en los contactos/entrevistas con los padres-madres. También, el desarrollo de las formas de colaboración establecidas entre los diferentes sectores de la comunidad educativa y la participación en las propuestas de mejora en los distintos ámbitos de actuación que se pueden establecer en las escuelas.

La edad parece determinar algunas de las competencias desarrolladas durante el Practicum. Los estudiantes con edades comprendidas entre 21 y 22 años consideran que tienen un mayor desarrollo de competencias vinculadas a las relaciones y formas de trabajo, mientras que los estudiantes de entre 23 y 24 años son los que más aplicación de conocimientos hacen en la práctica (tabla 7).

Tabla 7

Comparación de medias según edad de los estudiantes

\begin{tabular}{|c|c|c|c|c|c|c|}
\hline \multirow{5}{*}{ 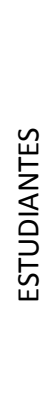 } & & $\begin{array}{l}\text { Media G1 } \\
\text { (SD) }\end{array}$ & Media G2 (SD) & Media G3 (SD) & Media G4 (SD) & $\begin{array}{l}\text { Dif. De } \\
\text { medias }\end{array}$ \\
\hline & El Practicum como asignatura (1) & $3.40(0.51)$ & $3.41(0.54)$ & $3.46(0.48)$ & $3.40(0.53)$ & $F=0.348$ \\
\hline & $\begin{array}{l}\text { Relaciones estudiantes-escuela- } \\
\text { universidad ( } 2 \text { ) }\end{array}$ & $2.89(0.61)$ & $3.07(0.64)$ & $3.04(0.60)$ & $3.02(0.66)$ & $\mathrm{F}=2.544 \mathrm{a}$ \\
\hline & Organización del Practicum (3) & $3.06(0.61)$ & $3.16(0.59)$ & $3.25(0.51)$ & $3.17(0.62)$ & $\mathrm{F}=2.457 \mathrm{a}$ \\
\hline & Global & $3.12(0.48)$ & $3.22(0.52)$ & $3.25(0.46)$ & $3.20(0.52)$ & $F=1.728$ \\
\hline \multicolumn{7}{|c|}{ G1= 20 o menos años, G2= entre 21 y 22 años, G3=entre 23 y 24 años, G4= 25 o más años } \\
\hline
\end{tabular}

En referencia a la titulación, los estudiantes de Infantil son los que consideran que han desarrollado más competencias a partir del Practicum (tabla 8). 
Tabla 8

Comparación de medias según

titulación de los estudiantes

\begin{tabular}{|c|c|c|c|c|}
\hline \multirow{5}{*}{ 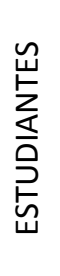 } & & Media G1 (SD) & Media G2 (SD) & $\begin{array}{l}\text { Dif. de } \\
\text { medias }\end{array}$ \\
\hline & Gestión de la dinámica del aula (1) & $3.44(0.50)$ & $3.38(0.54)$ & $F=1.758$ \\
\hline & Relaciones y formas de trabajo (2) & $3.03(0.64)$ & $2.98(0.61)$ & $F=0.772$ \\
\hline & Aplicación del conocimiento a la práctica (3) & $3.22(0.58)$ & $3.07(0.60)$ & $\mathrm{F}=8.438 \mathrm{c}$ \\
\hline & Global & $3.23(0.49)$ & $3.15(0.50)$ & $\mathrm{F}=3.510 \mathrm{a}$ \\
\hline
\end{tabular}

Además, el Practicum desarrollado durante el último curso del grado es el que más claramente contribuye al desarrollo de competencias (tabla 9).

Tabla 9

Comparación de medias según situación del Practicum

\begin{tabular}{|c|c|c|c|c|c|}
\hline \multirow{5}{*}{ 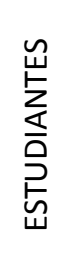 } & & $\begin{array}{l}\text { Media G1 } \\
\text { (SD) }\end{array}$ & $\begin{array}{l}\text { Media G2 } \\
\text { (SD) }\end{array}$ & $\begin{array}{l}\text { Media G3 } \\
\text { (SD) }\end{array}$ & $\begin{array}{l}\text { Dif. De } \\
\text { medias }\end{array}$ \\
\hline & Gestión de la dinámica del aula (1) & $3.40(0.52)$ & $3.43(0.52)$ & $3.40(0.44)$ & $F=0.145$ \\
\hline & Relaciones y formas de trabajo (2) & $3.00(0.63)$ & $3.00(0.64)$ & $3.016(0.57)$ & $\mathrm{F}=0.579$ \\
\hline & Aplicación de conocimiento a la práctica (3) & $3.16(0.58)$ & $3.14(0.60)$ & $3.07(0.71)$ & $F=0.173$ \\
\hline & Global & $3.019(0.49)$ & $3.19(0.51)$ & $3.22(0.50)$ & $F=0.038$ \\
\hline
\end{tabular}

Para los tutores de las escuelas las competencias más valoradas son: destrezas y habilidades sociales que fomentan un clima de aula que facilita el aprendizaje y la convivencia, actuación y reflexión desde la práctica y aplicación de procesos de interacción y comunicación en el aula.

Por el contrario, las competencias valoradas con un grado de adquisición menor son las que hacen referencia a: conocimiento práctico del aula y de la gestión de la misma (organización de los alumnos, del tiempo, de los espacios, participación en la acción tutorial y regulación en los procesos de interacción y comunicación de los grupos).

La titularidad de la escuela no es un factor determinante del desarrollo de competencias por parte de los alumnos. Sin embargo, podemos apreciar que existe una ligera diferencia significativa $(p<.1)$ en el caso de las competencias ligadas a la gestión de la dinámica del aula, donde las escuelas privadas/concertadas tienen una mayor contribución.

Los tutores de educación infantil consideran que el Practicum contribuye más claramente a desarrollar competencias vinculadas al aula.

Se reafirma el papel fundamental que juega el curso al que se vincula el Practicum. Como en el caso de los estudiantes, consideran que el Practicum asociado al último curso es el que más contribuye al desarrollo de competencias (tabla 10). 
Tabla 10

Comparación de medias según curso

\begin{tabular}{|c|c|c|c|c|c|c|}
\hline \multirow{5}{*}{ 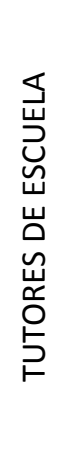 } & & $\begin{array}{l}\text { Media G1 } \\
\text { (SD) }\end{array}$ & $\begin{array}{l}\text { Media } \\
\text { G2 (SD) }\end{array}$ & Media G3 (SD) & $\begin{array}{l}\text { Media G4 } \\
\text { (SD) }\end{array}$ & $\begin{array}{l}\text { Dif. de } \\
\text { medias }\end{array}$ \\
\hline & $\begin{array}{l}\text { Gestión de la dinámica del } \\
\text { aula(1) }\end{array}$ & $3.00(0.60)$ & $3.00(0.55)$ & $3.01(0.61)$ & $3.28(0.53)$ & $\mathrm{F}=7.792 \mathrm{e}$ \\
\hline & $\begin{array}{l}\text { Relaciones y formas de } \\
\text { trabajo (2) }\end{array}$ & $\begin{array}{c}2.89 \\
(0.67) \\
\end{array}$ & $\begin{array}{c}2.92 \\
(0.69) \\
\end{array}$ & $2.96(0.63)$ & $3.12(0.57)$ & $F=2.918 b$ \\
\hline & $\begin{array}{l}\text { Aplicación de conocimientos } \\
\text { a la práctica(3) }\end{array}$ & $\begin{array}{c}3.08 \\
(0.57) \\
\end{array}$ & $\begin{array}{c}3.00 \\
(0.63) \\
\end{array}$ & $3.03(0.66)$ & $3.20(0.54)$ & $F=2.845 b$ \\
\hline & Global & $\begin{array}{c}2.99 \\
(0.55) \\
\end{array}$ & $\begin{array}{c}2.97 \\
(0.56) \\
\end{array}$ & $3.00(0.57)$ & $3.20(0.48)$ & $F=5.154 c$ \\
\hline
\end{tabular}

Y finalmente para los tutores de facultad las competencias valoradas son: adquisición de un conocimiento práctico del aula y de la gestión de la misma (organización de los alumnos, del tiempo, de los espacios, etc.), conocimiento y aplicación de procesos de interacción y comunicación en el aula y actuación y reflexión desde la práctica. Las dos primeras competencias con la puntuación más elevada coinciden con las dos competencias valoradas con un grado de adquisición más elevado de los estudiantes.

Por el contrario, las competencias valoradas con un grado de adquisición menor son: conocimiento de la acción tutorial con padres/madres y conocimiento de las formas de colaboración establecidas entre los diferentes sectores de la comunidad educativa y del entorno social. Estas dos competencias son también las valoradas con un grado de adquisición más bajo por parte de los estudiantes.

Cuando se hace una correlación con las variables que definen el perfil de los tutores de la facultad, hay que destacar que son los tutores con más experiencia los que consideran que el Practicum contribuye de forma más notable al desarrollo de competencias del alumnado. También, que la combinación de las modalidades intensivas y extensivas puede contribuir más a desarrollar las competencias para la gestión del aula.

\subsection{Competencias que más se trabajan}

Estudiantes y tutores de escuelas y facultades coinciden en afirmar que las competencias que más se trabajan son las relacionadas con el funcionamiento y gestión del aula.

La competencia "Conocimiento práctico del funcionamiento del aula» según los tutores de escuela, tutores de facultad y estudiantes se adquiere en el día a día de la estancia en la escuela, observando los procesos que tienen lugar en el aula, la intervención con el grupo-clase, la reflexión sobre la práctica, y trasladando lo trabajado y reflexionado a la memoria de prácticas. Cuanto más extensiva es la estancia en el centro, más se consolida esta competencia.

Te puedes hacer una idea, pero cuando lo ves en la realidad... A infantil es muy importante cómo está distribuida el aula, cómo aprovechas los espacios, etc. (ESC_15-EST_29).

Algunos tutores de escuela consideran que:

los alumnos en prácticas demuestran un buen conocimiento teórico de aspectos curriculares adquiridos en la facultad" (ESC_02-TUTESC_03 y 04),

y afirman que enseguida se implican adecuadamente. Sin embargo, otros tutores de escuela consideran que:

no conocen el funcionamiento del aula "(ESC_11-TUTESC_21 y 22), 
sobre todo en el caso de las escuelas singulares, como las Zona de Escuelas Rurales.

Para adquirir esta competencia se destaca la importancia de que los estudiantes tengan la oportunidad de intervenir en el aula: rutinas diarias (sobre todo en educación infantil), gestión de espacios, del tiempo, agrupamientos, organización de actividades, desarrollo de unidades didácticas, gestión de conflictos, etc.

Aprenden ayudando a hacer, leyendo, trabajando las rutinas, saliendo al patio, reflexionando sobre la práctica con el tutor, trabajando en las horas de exclusivas, asistiendo a las reuniones de trabajo, dando a conocer el currículo, etc. (ESC_16-TUTESC_31 y 32)

Pero más allá de estas actividades se destaca la importancia de la reflexión:

La práctica les permite reflexionar y ver puntos fuertes y puntos débiles. (ESC_13-TUTESC_25 y 26)

Por otra parte, los estudiantes valoran el hecho de estar en centros donde coincide la metodología trabajada en la universidad. La teoría dada en la universidad les ayuda a comprender la realidad del aula cuando el modelo que encuentran en las escuelas es paralelo; cuando la práctica no coincide con las expectativas, les crea confusión.

Si durante tres años de universidad escuchan que hay que trabajar por proyectos, hacer preguntas a los alumnos, etc. $y$, de repente, se encuentran en el aula con una enseñanza clásica, es aquí cuando se preguntan qué ha pasado. (ESC_02-TUTFAC_04)

Finalmente, los tutores de facultad y estudiantes echan en falta la posibilidad de asistir a reuniones de programación y de trabajo conjunto del profesorado.

Permitir que los alumnos puedan asistir a reuniones, a claustros, a tutorías con familias -con el permiso de la familia, por supuesto- es importante y se vincula a la docencia. Aparte del aula hay, una tarea mucho más amplia de reuniones y coordinaciones que han de ver. Es necesario que los alumnos estén enterados y lo conozcan. (ESC_09-TUTFAC_18)

En relación a la competencia "Gestión del aula», tutores de escuela, tutores de facultad y estudiantes coinciden en que se aprende a través de la intervención directa, en dinámicas del día a día o a través del diseño y aplicación de unidades didácticas. En este sentido, se indica que cuanto más tiempo se está en el aula, más se favorece su dominio:

Vivir las situaciones reales los hace desarrollar la competencia. (ESC_10-TUTESC_19 y 20)

Las prácticas ayudan a adquirir un mejor conocimiento sobre la gestión del tiempo en el aula y del grupo de alumnos. La intervención directa en el aula es una parte muy útil del Practicum. (ESC_02-EST_03 y 04)

También hay estudiantes y tutores que remarcan la importancia de observar y reflexionar sobre la intervención del profesorado de aula. Algunos tutores de escuela señalan algunas carencias en la intervención de los estudiantes: gestión del tiempo, cohesión interna de las actividades, saber estar.

No les cuesta situarse en el espacio, pero sí en el tiempo. Cuando llevan la unidad didáctica a la práctica, justamente el tiempo les viene grande. [...] Deberían pensar más allá de la actividad que programan: cuando programan su unidad didáctica, el reajustar no tiene cabida y sólo piensan en 'tengo que hacer'. La mirada de reajustar, de renunciar a algo previsto, de rehacer la actividad porque no ha salido bien, etc. Deben aprender a rehacer. (ESC_01-TUTESC_02)

En general, se remarca la necesidad de que los tutores de escuela den espacio para intervenir, pero que también sean los propios estudiantes los que tengan iniciativa 


\subsection{Competencias que menos se trabajan}

En general, tutores de facultad y estudiantes coinciden en indicar que la competencia relativa a «Conocimiento de la acción tutorial y los contactos con los padres-madres» es una competencia que falta desarrollar a nivel de estudios de Grado y en las prácticas.

Es un contenido que falta en los estudios de grado; no hay ninguna asignatura de cómo llevar a cabo una entrevista, una tutoría, etc. (ESC_01-EST_02)

Tú ves cómo se organizan para hacer las tutorías con los padres, pero no es una parte que se trabaje en el Practicum y en la Facultad tampoco se hace. (ESC_11-EST_21 y 22)

Algunos tutores de facultad consideran que desde la universidad habría que dar más relevancia y más contenido teórico a la acción tutorial. Sin embargo, el motivo principal por el que no se desarrolla durante las prácticas se relaciona con que la mayoría de estudiantes no tienen la posibilidad de asistir a reuniones con las familias (sobre todo las individuales), por considerar las escuelas que es un momento para hacer un traspaso de información confidencial donde los estudiantes no deben tener acceso. También se considera que, dado el tiempo de presencia de los estudiantes, no se establece un grado de compromiso suficiente con la escuela para permitir acceder a esta información.

En las entrevistas con los padres, a veces, salen cosas muy íntimas y no sé si la presencia del alumno de prácticas podría incluso coaccionar a los mismos. (ESC_02-TUTESC_03)

Tutores de facultad y estudiantes señalan que la relación que puedan establecer los estudiantes con las familias depende de cada centro y tutor. Los tutores de facultad consideran que habría que incidir en que el alumno intervenga en otras situaciones fuera del aula; sin embargo, los tutores de escuela consideran que hay otras acciones en las que los estudiantes pueden desarrollar esta competencia: reuniones de inicio de curso, en la recogida de los alumnos, tutorías de aula con los niños, actividades abiertas a las familias, vigilancia de los patios, etc.

Los estudiantes que han podido asistir a actividades de acción tutorial valoran muy positivamente la experiencia, al considerarla como una oportunidad para formarse en aspectos que en la Facultad no surgen.

Por otra parte se subraya que los estudiantes deben ser considerados como una parte del equipo de maestros y se les debería dar la oportunidad de asistir a reuniones de claustro de profesores, de consejo escolar, de reuniones con familias, etc., siempre que sea posible.

Nosotros no vetamos las reuniones, en general. Sí, cuando se trata de reuniones donde la confidencialidad o la seguridad del alumno son importantes (GD_DIR-01_UAB)

En los grupos de discusión, se argumenta que la solución pasa por incluir la acción tutorial como parte del plan de prácticas, tanto desde la Facultad como desde las escuelas, y que el tema de la protección de la información se puede resolver si los estudiantes firman una carta de compromiso de confidencialidad.

Si la Facultad entiende que el ámbito de la acción tutorial es bastante importante [...], debería marcarlo como una condición del Plan de Prácticas. Si desde la Facultad atamos las expectativas de aprendizaje del alumno y las comunicamos en el centro, probablemente no serían tanto aprender por tentativa, como les está pasando: que cuando vas a un centro no hay Claustro o no me dejan entrar en el Claustro, que voy a otro y no a las tutorías... vamos por tentativa y no está sistematizado y debería formar parte del interés de las dos partes de una manera convenida y planificada. (GD_ADM_UdL) 


\section{Discusión y Conclusiones}

El contacto con la realidad es una parte imprescindible de los planes de estudios de los Grados de Infantil y Primaria y las prácticas permiten a los estudiantes desarrollar las competencias profesionales (Paredes-Labra y al, 2016; Arnold, Groschner y Hascher, 2014; Egido y López, 2016; Rodríguez-Gómez, Armengol y Meneses, 2017; ). Está claro que el modelado, la observación, la reflexión y las intervenciones en el aula de los centros de prácticas favorecen el desarrollo de la competencia de conocimiento práctico del aula y de su gestión. Cuanto más prolongada y continuada sea la estancia, más elevado podrá ser su grado de consecución. El estudiante desarrolla esta competencia en la escuela gracias a la implicación del tutor del centro quien asume un papel de guía y de apoyo psicológico y emocional para poder hacer frente a los retos de la práctica en el aula (Jaspers, Meijer, Prins y Wubbels, 2014; Sorensen, 2014; Gairín, Díaz-Vicario, del Arco y Flores , 2019).

Estudiantes, tutores de facultad y tutores de escuela valoran que el desarrollo y aplicación de competencias para el ejercicio de la profesión docente durante el Practicum es suficiente, siendo los informantes del grado de Infantil los que más valoran la adquisición de competencias en el Practicum. Paralelamente, hay que subrayar que los tutores noveles de las escuelas y los experimentados de las facultades coincidan en afirmar que la contribución del Practicum en el desarrollo competencial del futuro docente es determinante para una buena formación docente.

Se constata que la adquisición de competencias profesionales está asociada al rol activo que se le otorgue al estudiante de prácticas, lo que les ayuda a construir progresivamente su identidad profesional como educadores (Ralph y Walker, 2014).

Se confirma (Zabalza, 2004) que las prácticas no deben vincularse, al menos de forma prioritaria, al empleo sino a la formación y que la discusión pública privada es un debate estéril. Nuestro estudio constata que no existen diferencias en relación a la adquisición de competencias en función de la titularidad del centro donde estas se hayan realizado.

El calendario de prácticas utilizado en sus diversas variaciones tampoco se manifiesta como un elemento decisivo en la adquisición de competencias. Estudios como el de Eurydice (2005) y Zabalza (2004) alertan que su aprovechamiento estará en consonancia con la coherencia de la actividad práctica que se realiza en las escuelas y el resto de los componentes del programa de formación. Temporizaciones del Practicum intercaladas a lo largo del programa formativo ayudarán al fortalecimiento del desarrollo de las materias, si se hace uso de las experiencias desarrolladas. Queda evidenciada la necesidad de una buena planificación, tal y como argumenta Zabalza (2004) las prácticas por ellas mismas no serán beneficiosas si no están debidamente organizadas y diseñadas para que se produzca una interrelación entre la teoría y la práctica.

El presente estudio deja claro, por otra parte, que las actividades vinculadas con la acción tutorial dan poco margen de participación a los estudiantes, sobre todo en lo referente al contacto con las familias y no solo por motivos de confidencialidad y respeto a las mismas. Los tutores de escuela consideran que se podría paliar esta circunstancia si los estudiantes se implicaran en otras actividades (reuniones de inicio de curso, actividades de tutoría en aula, sesiones abiertas a las familias, etc.). Se entiende que el escaso desarrollo de las habilidades relacionadas con la tutoría también se debe al escaso desarrollo que tiene este tema en las asignaturas del Grado y en los programas de Practicum, sugiriendo buscar un equilibrio e incidir en la acción tutorial y en la importancia de que los estudiantes puedan participar e implicarse en actividades más allá del aula. El escaso desarrollo de las habilidades relacionadas con la tutoría contrasta con la alta consideración de las habilidades relacionadas con el conocimiento práctico en lo organizativo y didáctico del funcionamiento del aula. 
Todo ello nos lleva a subrayar la importancia del Practicum en la formación de maestros como una experiencia que sitúa al estudiante de magisterio ante una realidad compleja, poliédrica y dispar (González, Alba, Arcos, 2020) que va más allá de la trasmisión de conocimientos (Martínez y Fernández, 2018; Susinos y Sáiz, 2016). Deberán adquirir competencias que ayuden a definir la identidad profesional del maestro (Leite, Cortés, Rivas, 2016) en consonancia con las variables políticas, sociales, culturales y científicas de la realidad donde desarrollan el ejercicio profesional educativo y que supone no basarse exclusivamente en la transmisión de los contenidos. Como señalan González, Leite y Rivas (2018) el Practicum no es una asignatura donde el estudiante es receptáculo de saberes prácticos en el aula, sino que debe convertirse en una experiencia de transformación, construcción, re-construcción desde la praxis de la identidad docente desde todas sus dimensiones de acción.

\section{Referencias bibliográficas}

ANECA (2004). Libro Blanco sobre titulación de Grados de Magisterio de 2004. España. Vol. 1,2

Arnold, Karl-Heinz ; Gröschner, Alexander ; Hascher, Tina (2014). Experiencias pedagógicas de campo en la formación del profesorado: Introducción al área de investigación. En: Arnold, Karl-Heinz ; Gröschner, Alexander ; Hascher, Tina (eds.) Experiencias de campo pedagógicas en la formación del profesorado / Schulpraktika in der Lehrerbildung (págs. 9-26). Münster: Waxmann

Cabrerizo, J., Rubio, M. J., I Castillo, S. (2010). El prácticum en los grados de pedagogía, de magisterio y de educación social. Madrid: Pearson Educación

Coller, X. (2000). Estudio de casos. Madrid: Centro de Investigaciones Sociológicas.

Creswell, J. W. (2009). Editorial: Mapping the field of mixed methods research. Journal of Mixed Methods Research, 3(2), 95-108.

Egido, I., y López, E. (2016). Condicionantes de la conexión entre la teoría y la práctica en el Prácticum de Magisterio: Algunas evidencias a partir de TEDS-M. Estudios sobre Educación, 30, 217- 237. http://doi.org/10.15581/004.30.217-237

EURYDICE (2002). Las Competencias Clave: Un concepto en expansión dentro de la educación general obligatoria. Bruselas: Eurydice.

EURYDICE (2004). La profesión docente en Europa: Perfil, tendencias y problemática. Informe IV: El atractivo de la profesión docente en el siglo XXI. Educación Secundaria Inferior General. Madrid: Secretaría General Técnica C.I.D.E.

EURYDICE (2005). La profesión docente en Europa: Perfil, tendencias y problemática. Informe anexo: Reformas de la profesión docente: análisis histórico (1975-2002). Educación Secundaria Inferior General. Madrid: Secretaría General Técnica C.I.D.E.

Gairín, J., ArmengoL, C., del Arco, I., Muñoz, J.L., I Rodríguez-Gómez, D. (2013). Prácticas curriculares en la formación universitaria de los futuros profesionales. Revista Practicum , 4 (1), 19-36. ISSN: 2530-4550

Gairín, J., Díaz-Vicario, A., del Arco, I. y Flores, O. (2019). Efecto e impacto de las prácticas curriculares de los Grados de Educación Infantil y Primaria: la perspectiva de estudiantes, tutores y coordinadores. Educacion XX1. DOI: $10.5944 /$ educXX1.21311

Gálvez, I. E., \& Martín, E. L. (2016). Condicionantes de la conexión entre la teoría y la práctica en el Prácticum de Magisterio: Algunas evidencias a partir de TEDS-M. Estudios sobre Educación, 30, 217-237. https://doi.org/10.15581/004.30.217-237 
González, P. C., Alba, B. G., \& Arcos, D. P. (2020). Visiones del alumnado y el sentido del prácticum en educación primaria. Revista interuniversitaria de formación del profesorado, 34(2), 275-298. https://doi.org/10.47553/rifop.v34i2.79613

González, B., Leite, A. E., y Rivas, J. I. (2018). La indagación educativa como herramienta de transformación social. En V. Martín Solbes y M. T. Castilla (Coords.), Educación, derechos humanos y responsabilidad social (pp.127142). Barcelona: Octaedro. ISBN: 978-84-17667-00-9

González Sanmamed, M. (2001). ¿Qué se aprende en el Practicum? ¿Qué hemos aprendido sobre el Practicum? En L. Iglesias, M. Zabalza, A. I. Cid, M. Raposo (Coords.), Desarrollo de Competencias Personales y Profesionales en el Practicum. VI Symposium Internacional sobre el Practicum. Lugo: Unicopia.

Hernández Sampieri, R., Fernández-Collado, C., Baptista, P. (2008). Metodología de la investigación. México: McGraw-Hill.

Jaspers, W. M., Meijer, P. C., Prins, F., \& Wubbels, T. (2014). Mentor teachers: Their perceived possibilities and challenges as mentor and teacher. Teaching and teacher education, 44, 106-116. https://doi.org/10.1016/j.tate.2014.08.005

Leite, A., Cortés, P., y Rivas, J. I. (2016). Narrativa y creatividad en la Universidad: ¿es posible transitar otros caminos en la enseñanza y el aprendizaje? En P. Cortés-González y M. J. Márquez. Creatividad, Comunicación y Educación. Más allá de las fronteras del saber establecido (pp. 151-164). Málaga: UMA Editorial. DOI: 10.17583/qre.2017.2590

Marhuenda, F., Bernad, J. C., Navas, A. (2010). Las prácticas en empresa como estrategia de enseñanza e inserción laboral: las empresas de inserción social. Revista de Educación, 351, 139-161.

Martínez, J. B., y Fernández, E. (2018). Ecologías del aprendizaje. Educación expandida en contextos múltiples. Madrid: Morata.ISBN: 978-84-7112-874-4

Miles, M. B., Huberman, M. (1994, 2ae ed.). Qualitative Data Analysis: An Expanded Sourcebook. Thousand Oaks, CA: Sage.

Miles, M., Huberman, M., Saldaña, J. (2014, 3a ed.). Qualitative Data Analysis. Thousand Oaks, CA: Sage

Miller, R. (1981). Simultaneous Statistical Inference (2 ed.). New York: Springer-Verlag

OCDE (2006). Attracting, Developing and Retaining Effective Teachers - Final Report: Teachers Matter. París: OECD

OCDE (2009). Creating Effective Teachingand Learning Environments. Teaching and Learning International Survey (TALIS), Paris: OCDE. Recuperado 05/10/2016 de http://www.oecd.org/education/school/43023606.pdf

ORDEN ECl/3857/2007, de 27 de diciembre, por la que se establecen los requisitos para la verificación de los títulos universitarios oficiales que habiliten para el ejercicio de la profesión de Maestro en Educación Primaria. Boletín Oficial del Estado, 312, 53747-53750. Recuperado 06/08/2016 de https://www.boe.es/diario_boe/txt.php?id=BOE-A-2007-22449

OIT/UNESCO (2006): Comité OIT/UNESCO de expertos sobre la aplicación de la Recomendación relativa al Personal Docente. Informe. Novena reunión (Ginebra, OIT).

OIT/UNESCO (2008): Recomendación relativa al Personal Docente (1966). Paris, UNESCO. 
Paredes-Labra, J; Esteban-Moreno, R y Fernández-Prieto, MS (2016): El Prácticum de Maestro en las voces de sus tutores. Balance del plan 2010 en la UAM. Revista Complutense de Educación. 27 (1) (2016) 161-178 DOI: https://doi.org/10.5209/rev_RCED.2016.v27.n1.45673.

Ralph, E. G. y Walker, K. D. (2014). Mentorship in the Prácticum: Post-Interns'Perspectives. International Journal of Humanities and Social Science, 4(8), 1-10. Recuperado 12/07/2016 de http://ijhssnet.com/journals/Vol_4_No_8_June_2014/1.pdf

Rodríguez-Gómez, D., Armengol, C., \& Meneses, J. (2017). La adquisición de las competencias profesionales a través de las prácticas curriculares de la formación inicial de maestros. Revista de Educación, 376. AbrilJunio 2017, pp. 229-251. DOI: 10.4438/1988-592X-RE-2017-376-350

Sandín, M. (2003). Investigación cualitativa en educación. Fundamentos y tradiciones. Madrid: McGraw-Hill. ISBN: 84-481-3779-5

Sorensen, P. (2014). Collaboration, dialogue and expansive learning: The use of paired and multiple placements in the school Practicum. Teaching and teacher education, 44, 128-137. https://doi.org/10.1016/j.tate.2014.08.010

Susinos, R., y Sáiz (2016). Los problemas pedagógicos son mis aliados. El Prácticum como un proceso de reflexión e indagación colaborativa. Revista de Investigación en Educación, 1(14), 5-13.

TALIS (2013). Estudio internacional sobre la enseñanza y el aprendizaje. Informe español. Recuperado 12/05/2016 de http://www.mecd.gob.es/inee/Ultimos_informes/TALIS-2013.html

Toothaker, LE (1991). Múltiples comparaciones para investigadores . Publicaciones Sage, Inc.

Tomarken, A. J.; Serlin, R. C. (1986). Comparison of ANOVA alternatives under variance heterogeneity and specific noncentraly structures. Psychological Bulletin. Vol. 99, pàg. 90-99. DOI: 10.1037 / 0033-2909.99.1.90

Villar, L.M. (1998): Formación práctica de los maestros. En A. Rodríguez, E. Sanz y M.V Sotomayor (coords): La formación de los maestros en los Países de la Unión Europea. Madrid, Narcea.

Zabalza, M. (2004). Condiciones para el desarrollo del prácticum. Profesorado, revista de currículum y formación del profesorado, $8(2), 1-21$.

Zabalza, M. (2011). El prácticum en la formación universitaria: estado de la cuestión. Revista de educación, 354, 21-43. 